\title{
Carbon Nanotube Scaffolds Tune Synaptic Strength in Cultured Neural Circuits: Novel Frontiers in Nanomaterial-Tissue Interactions
}

\author{
Giada Cellot, ${ }^{1}$ Francesca Maria Toma, ${ }^{2}$ Zeynep Kasap Varley, ${ }^{3}$ Jummi Laishram, ${ }^{1}$ Ambra Villari, ${ }^{1}$ Mildred Quintana, ${ }^{2}$ \\ Sara Cipollone, ${ }^{2}$ Maurizio Prato, ${ }^{2}$ and Laura Ballerini ${ }^{1}$ \\ ${ }^{1}$ Life Science Department, B.R.A.I.N., University of Trieste, I-34127 Trieste, Italy, ${ }^{2}$ Department of Pharmaceutical Sciences, University of Trieste, I-34127 \\ Trieste, Italy, and ${ }^{3}$ Neurobiology Sector, International School for Advanced Studies (SISSA), I-34136 Trieste, Italy
}

\begin{abstract}
A long-term goal of tissue engineering is to exploit the ability of supporting materials to govern cell-specific behaviors. Instructive scaffolds code such information by modulating (via their physical and chemical features) the interface between cells and materials at the nanoscale. In modern neuroscience, therapeutic regenerative strategies (i.e., brain repair after damage) aim to guide and enhance the intrinsic capacity of the brain to reorganize by promoting plasticity mechanisms in a controlled fashion. Direct and specific interactions between synthetic materials and biological cell membranes may play a central role in this process. Here, we investigate the role of the material's properties alone, in carbon nanotube scaffolds, in constructing the functional building blocks of neural circuits: the synapses. Using electrophysiological recordings and rat cultured neural networks, we describe the ability of a nanoscaled material to promote the formation of synaptic contacts and to modulate their plasticity.
\end{abstract}

\section{Introduction}

Ongoing efforts in regenerative medicine require the development of synthetic extracellular scaffolds customized to provide unique microenvironments to tissue-specific cell types (Place et al., 2009). Traditionally, this can be achieved by tissueengineering scaffolds enriched by molecular elements that mimic matrix features (Place et al., 2009). A variety of nanostructures, such as 3D bioactive scaffolds, have been investigated for tissue engineering applications (Cui et al., 2010). More recently, several studies took advantage of more basic chemistry to obtain structurally simple materials, where the topography and nanoscale dimensions are crucial to promote native protein adsorption, eventually mediating cell interactions (Woo et al., 2003; Place et al., 2009). Ultimately, characterizing the relevance of nanoscaled extracellular features in directing specific cellular processes has

Received March 15, 2011; revised July 14, 2011; accepted July 18, 2011.

Author contributions: M.P. and L.B. designed research; G.C., F.M.T., Z.K.V., J.L., and A.V. performed research;S.C., M.Q., and M.P. contributed unpublished reagents/analytic tools; G.C., F.M.T., Z.K.V., J.L., A.V., M.P., and L.B. analyzed data; G.C. and L.B. wrote the paper.

Financial support from the EU (NEURONANO-NMP4-CT-2006-031847 and CARBONANOBRIDGE-ERC-2008227135 , to L.B. and M.P.) is gratefully acknowledged. We are especially grateful to E. Cherubini for his helpful comments and for his critical revision of the manuscript. We are grateful to P. Zacchi for helping in the immunofluorescence procedures, F. Bonaccorso and G. Privitera for conductivity measurements and helpful discussions, L. Masten for assistance with tissue cultures, R. Rauti for electrophysiological measurements, and C. Gamboz for TEM procedures.

The authors declare no competing financial interests.

Correspondence should be addressed to either of the following: Dr. Laura Ballerini, Life Science Department, Center for Neuroscience B.R.A.I.N., University of Trieste, via Giorgeri 1, 34127, Trieste, Italy, E-mail: Iballerini@units.it; or Prof. Maurizio Prato, Center of Excellence on Nanostructured Materials, Department of Pharmaceutical Sciences, University of Trieste, Piazzale Europa 1,34127 Trieste, Italy, E-mail: prato@units.it.

DOI:10.1523/JNEUROSCI.1332-11.2011

Copyright $\odot 2011$ the authors $\quad 0270-6474 / 11 / 3112945-09 \$ 15.00 / 0$ set the stage for nanoscience and tissue engineering research synthesis (Dvir et al., 2011).

Nerve tissue engineering, which aims at rebuilding lesioned CNS circuits (Plant et al., 1997; Geller and Fawcett, 2002; Orive et al., 2009), has increasingly involved nanotechnology for the development of supermolecular architectures to sustain and promote neural regeneration following injury (Silva, 2006, 2009). For example, a pioneering study used nanofibers to provide optic tract regeneration in hamsters (Ellis-Behnke et al., 2006). More recently, complementary strategies emerged, leading to hybrid systems with macromolecules and/or inorganic components, such as carbon nanotubes, whose potential applications require further investigations (Cui et al., 2010; Dvir et al., 2011).

Due to the potential role of the interactions between nanomaterials and neurons in guiding the design of future materials for engineering electrically propagated tissues (Dvir et al., 2011), we investigated the impact of carbon nanotubes in the assembly of neuronal circuits in culture. The size, high electrical conductivity, and large surface area of carbon nanotubes favor their interactions with distal dendrites, promoting the emergence of novel nanoengineered neural devices (Cellot et al., 2009; Silva, 2009).

Carbon nanotubes have been at the forefront of nanotechnology due to their unique features, which allow the development of a variety of miniaturized devices with remarkable properties. Single-walled and multiwalled carbon nanotubes (SWCNTs and MWCNTs, respectively) have attracted tremendous attention as potential scaffolds for reestablishing the intricate connections between neurons (Mattson et al., 2000; Hu et al., 2005; Lovat et al., 2005; Ni et al., 2005; Galvan-Garcia et al., 2007; Cellot et al., 2009); they are an ideal material for long-term neural implants, as they enhance electrical recording of neurons in culture and in 
A
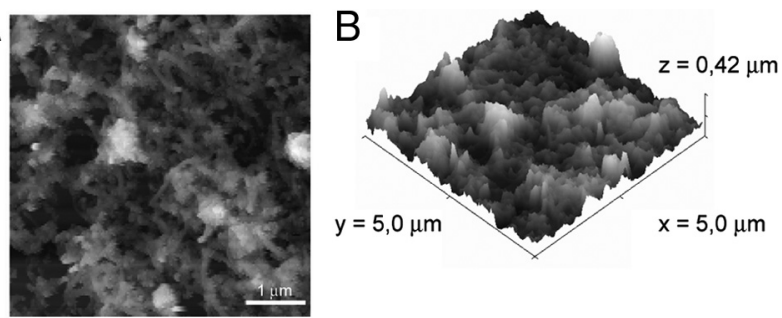

C
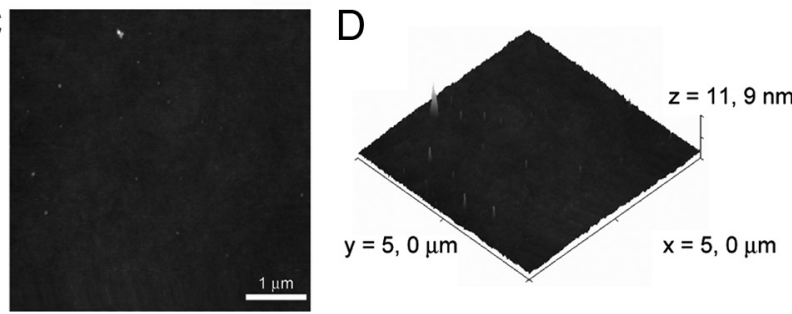

Figure 1. MWCNT and control substrates show different roughness. $A-D, A F M$ images of glass substrates coated with MWCNT $(\boldsymbol{A}), 3 \mathrm{D}$ height profile of the image in $\boldsymbol{A}(\boldsymbol{B})$, bare glass $(\boldsymbol{C})$, and $3 D$ height profile of the image in $\boldsymbol{C}(\boldsymbol{D})$. The calculated roughness of coated substrates is $30.7 \pm 4.0 \mathrm{~nm}$, whereas the calculated roughness of bare glass is $0.3 \pm 0.02 \mathrm{~nm}$.

living animals (Keefer et al., 2008; Shoval et al., 2009) by reducing the impedance between devices and cell membranes (Kotov et al., 2009).

In carbon nanotube-neuron hybrid networks, the cultured neurons always display a boost in signal transmission, which is detected as an increase in the frequency of synaptic events (Lovat et al., 2005; Mazzatenta et al., 2007). We recently reported that direct nanotube-substrate interactions with the membranes of neurons can affect single-cell activity (Cellot et al., 2009). Here we use these unique material properties to promote, by carbon nanotube supporting platforms, network connectivity and synaptic plasticity in mammalian cortical circuits.

\section{Materials and Methods}

Synthesis of MWCNTs

MWCNTs 20-30 nm (Nanostructured \& Amorphous Materials), used as received, were prepared as described previously (Lovat et al., 2005).

Thermal gravimetric analysis (TGA) Q500 (TA Instruments) was used to record TGA analyses under $\mathrm{N}_{2}$ or under air, by equilibrating at $100^{\circ} \mathrm{C}$, and following a ramp of $10^{\circ} \mathrm{C} / \mathrm{min}$ up to $1000^{\circ} \mathrm{C}$. In the case of defunctionalized MWCNTs, the material was analyzed after exposing to high temperature $\left(350^{\circ} \mathrm{C}\right)$ under $\mathrm{N}_{2}$. TGA was routinely repeated to test every new MWCNT batch.

MWCNT thin film was characterized by sheet resistance measurements obtained using a Jandel four tips probe. Then thickness of the film was measured by atomic force microscopy (AFM) and conductivity of the material was extracted. MWCNT-coated glass substrate AFM images were analyzed by Gwyddion software (free scanning probe microscopy data analysis software) and average roughness calculated as Rms was derived (Fig. 1).

\section{Electron microscopy}

Transmission electron microscopy (TEM) analyses were performed on a TEM Philips EM208, using an accelerating voltage of $100 \mathrm{kV}$. About $1 \mathrm{mg}$ of compound was dispersed in $1 \mathrm{ml}$ of solvent. Then, one drop of this solution was deposited on a TEM grid (200 mesh, nickel, carbon only). Scanning electron microscopy (SEM) measurements were performed with a Zeiss Supra microscope. SEM analysis of MWCNTs was routinely performed to test every new batch. AFM measurements were registered with a Veeco V in tapping mode. Phosphorus (n)-doped silicon tips with a resonance frequency of $273-325 \mathrm{kHz}$ and a constant force of $20-80$ $\mathrm{N} / \mathrm{m}$ were used.

\section{Primary cultures}

Hippocampal neurons were obtained from postnatal rat pups (of either sex) as previously reported (Lovat et al., 2005), cells were plated on growth-promoting peptide-free glass (control cultures) or on MWCNTtreated glass (CNT cultures) coverslips, to investigate growth interfaces that differed only in terms of electrical conductivity and nanoscaled structure (Cellot et al., 2009). Cultured cells were used for experiments at $8-14 \mathrm{~d}$ in vitro. Neurons were seeded on peptide-free control and MWCNT substrates with a standard amount of cells (quantified in total $130,000 \pm 15,000$ cells; in a sample of $n=4$ cultures). Even in the absence of exogenous layers of growth-promoting peptides, in our culturing conditions cells attached to the substrates (both glass and MWCNT) within $1 \mathrm{~h}$ from the seeding, and then they grew, developing typical neurite specializations, with a morphology similar to that detected in poly-Dlysine (Mazzatenta et al., 2007) or RADA16 (Cellot et al., 2009) substrates. To quantify neuronal density (see also Lovat et al., 2005), cultures (control and CNT) were immunostained following the procedure previously described (Avossa et al., 2003). Briefly, cultures were fixed with $4 \%$ paraformaldehyde in PBS (20 min), then, upon washout in PBS, incubated in blocking solution and subsequently incubated with rabbit polyclonal antibody against $\beta$ tubulin III (1:50 dilution; Sigma-Aldrich). Upon washout in PBS, cultures were incubated with the secondary goat anti-rabbit Alexa Fluor 594 (1:500, Invitrogen). We sampled two culture series (5-7 fields for each slide), and we found similar densities in $\beta$-tubulin III-positive cells in the different conditions of growth (1180 \pm 140 cells $/ \mathrm{mm}^{2} ; n=27$ fields, in control, and $1230 \pm 231$ cells $/ \mathrm{mm}^{2} ; n=$ 26 fields, in CNT; 2 culture series, $n=2$ coverslips, for each group).

For experiments involving chronic treatments, cultures were incubated at day 4 in vitro with a medium containing $1 \mu \mathrm{M}$ tetrodotoxin (TTX). At day 8 or 9 (after $4-5 \mathrm{~d}$ of incubation), the medium was replaced with a fresh one, without the blocker, for $2 \mathrm{~h}$ before electrophysiological recordings. Control cultures were subjected to the same medium changes without addition of the blocker. In our previous work, we have confirmed the chemical stability of TTX under these experimental conditions (Galante et al., 2000).

\section{Electrophysiology}

Pair recordings. Paired whole-cell recordings were obtained with pipettes (4-7 M $\Omega$ ) containing $105 \mathrm{~mm} \mathrm{~K}$ gluconate, $30 \mathrm{~mm} \mathrm{KCl,} 10$ mM HEPES, 4 mm MgATP, 0.3 mM GTP, pH 7.35. The external solution contained the following: $150 \mathrm{~mm} \mathrm{NaCl}, 4 \mathrm{~mm} \mathrm{KCl}, 1 \mathrm{~mm} \mathrm{MgCl}_{2}, 2 \mathrm{~mm} \mathrm{CaCl}_{2}, 10 \mathrm{~mm}$ HEPES, $10 \mathrm{~mm}$ glucose, $\mathrm{pH}$ 7.4. For experiments where the external calcium $\left(\mathrm{Ca}^{2+}\right)$ was changed, the $\mathrm{Ca}^{2+} / \mathrm{Mg}^{2+}$ ratio was adjusted accordingly. All experiments were performed at $18-22^{\circ} \mathrm{C}$. Data were collected using two amplifiers. For current-clamp recordings, we used an Axoclamp 2 series amplifier (Molecular Devices) under bridge balance mode (that was continuously monitored and adjusted). At $-60 \mathrm{mV}$ resting membrane potential ( $\leq 0.03 \mathrm{nA}$ negative current injection), we elicited in the presynaptic cell action potentials (APs) by injecting short $(4 \mathrm{~ms}$ ) square current pulses $(0.5-1 \mathrm{nA})$.

The postsynaptic cell was voltage clamped, usually at $-58 \mathrm{mV}$ holding potential (not corrected for liquid junction potential, that was $14 \mathrm{mV}$ ) and data were acquired with an EPC-7 amplifier (List); the uncompensated value for series resistance was $<8-11 \mathrm{M} \Omega$.

Current and voltage clamp responses were digitized at $10-20 \mathrm{kHz}$ with the pCLAMP 10 software (Molecular Devices) and stored for further analysis.

Neuronal passive properties were routinely measured in voltage and/or current clamp, and were comparable between control and CNT sampled cells. On average, cell capacitance was $55 \pm 3 \mathrm{pF}(n=45$, control $)$ and $59 \pm 4 \mathrm{pF}(n=31, \mathrm{CNT})$, cell input resistance was $523 \pm 44$ $\mathrm{M} \Omega(n=45$, control $)$ and $600 \pm 54 \mathrm{M} \Omega(n=31, \mathrm{CNT})$, and resting membrane potential was $-46 \pm 2 \mathrm{mV}(n=38$, control $)$ and $-45 \pm 4$ $\mathrm{mV}(n=28, \mathrm{CNT})$.

These values are in agreement either with those measured from neurons cultured in the presence of growth-promoting peptides (RADA16, see Cellot et al., 2009) and with those reported in the literature (Potthoff and Dietzel, 1997; Bolton et al., 2000; Kasap Varley et al., 2011). Upon TTX treatment, neurons exhibit comparable values for capacitance 
Table 1. Properties of CNT pairs

\begin{tabular}{lll}
\hline & Control ( $n=19$ pairs) & CNT $(n=38$ pairs) \\
\hline Presynaptic AP amplitude (mV) & $113 \pm 5$ & $104 \pm 4$ \\
Postsynaptic PSC latency (ms) & $2.1 \pm 0.3$ & $2.3 \pm 0.2$ \\
Postsynaptic PSC rise time (ms) & $3.5 \pm 0.4$ & $4.5 \pm 0.4$ \\
Postsynaptic PSC decay time (ms) & $36 \pm 2$ & $33 \pm 1$ \\
\hline
\end{tabular}

Properties of the presynaptic induced APs and of the evoked postsynaptic unitary GABAergic PSCs are compared between control and CNT neuronal pairs.

Table 2. Properties of untreated and TTX treated CNT pairs

\begin{tabular}{lcc}
\hline & $\begin{array}{c}\text { TTX-untreated CNT } \\
(n=13 \text { pairs) }\end{array}$ & $\begin{array}{c}\text { TTX-treated CNT } \\
(n=12 \text { pairs })\end{array}$ \\
\hline Presynaptic AP amplitude (mV) & $107 \pm 7$ & $104 \pm 9$ \\
Postsynaptic PSC latency (ms) & $2 \pm 0.3$ & $2 \pm 0.3$ \\
Postsynaptic PSC amplitude (pA) & $76 \pm 25$ & $97 \pm 22$ \\
Postsynaptic PSC rise time (ms) & $3.4 \pm 0.4$ & $2.7 \pm 0.3$ \\
Postsynaptic PSC decay time (ms) & $36 \pm 2$ & $34 \pm 3$ \\
\hline
\end{tabular}

Properties of the presynaptic induced APs and of the evoked postsynaptic unitary GABAergic PSCs are compared between untreated and TTX-treated CNT neuronal pairs.

Table 3. Carbon nanotubes boost network activity

\begin{tabular}{llll}
\hline & $\begin{array}{l}\text { AMPA receptor-mediated } \\
\text { PSC frequency }(\mathrm{Hz})\end{array}$ & $\begin{array}{l}\mathrm{GABA}_{\mathrm{A}} \text { receptor-mediated } \\
\text { PSC frequency }(\mathrm{Hz})\end{array}$ & $\begin{array}{l}\text { Total PSC } \\
\text { frequency }(\mathrm{Hz})\end{array}$ \\
\hline Control $(n=7)$ & $0.22 \pm 0.08$ & $0.16 \pm 0.04$ & $0.38 \pm 0.11$ \\
CNT $(n=6)$ & $1.07 \pm 0.41^{*}$ & $0.94 \pm 0.27^{*}$ & $2.05 \pm 0.41^{*}$
\end{tabular}

Hippocampal networks grown on MWCNT substrates display a typical increase in PSC frequency, in both glutamatemediated and GABA-mediated events $\left({ }^{*} p<0.05\right)$.

$(58 \pm 5 \mathrm{pF} n=26)$, for the input resistance ( $597 \pm 59 \mathrm{M} \Omega, n=26)$, and for the resting membrane potential $(-45 \pm 3 \mathrm{mV} ; n=16)$.

Monosynaptic connections were recognized by their short latency $(<3$ ms, Pavlidis et al., 2000), measured between the peak of the elicited presynaptic AP and the onset of the postsynaptic current (PSC) response obtained in voltage clamp (Tables 1,2 ). We excluded from this study all recordings where clear bisynaptic connections were present and those where ongoing spontaneous activity was occasionally superimposed to the evoked PSCs.

We identified the different populations of evoked PSCs on the basis of their kinetic properties, reverse potential, and pharmacology (Galante et al., 2000). In fact, glutamatergic AMPA receptor-mediated PSCs display fast decay $(\tau=8 \pm 1.3 \mathrm{~ms}, n=8)$ inverted polarity around $0 \mathrm{mV}$ holding potential $(n=3)$ and were further blocked by application of $10 \mu \mathrm{M}$ CNQX $(n=2)$. GABAergic PSCs display slow decay $(\tau=34 \pm 1 \mathrm{~ms}, n=$ 57) inverted polarity at $-40 \mathrm{mV}$ holding potential, which is close to the Nernst value for $\mathrm{Cl}^{-}$reverse potential in our experimental conditions $(n=4)$, and were fully abolished by administration of $5 \mu \mathrm{M}$ gabazine $(n=11)$

In both culture groups (control and CNT), the large majority $(>85 \%$ ) of monosynaptically coupled pairs displayed evoked currents mediated by the $\mathrm{GABA}_{\mathrm{A}}$ receptors; we usually detected only a minority $(\leq 15 \%$ in control and in CNT) of AMPA receptor-mediated monosynaptic PSCs. There are discordant reports (Basarsky et al., 1994; Bi and Poo, 1998) on the occurrence of GABAergic or glutamatergic monosynaptic connections in pair recordings in dissociated hippocampal cultures. These discrepancies may involve differences in the culturing procedures, such as the animals' age, the cellular density, or the medium composition. However, our results, which indicate the larger occurrence of inhibitory monosynaptic connections (when closely located neurons are simultaneously patch clamped), are in agreement with other reports (Segal, 1983; Melnick et al., 1999). In addition, when recording spontaneous activity, the majority of spontaneous PSCs were reported to be glutamatergic in our study (Table 3); the higher occurrence of GABAergic monosynaptic connections is apparently due to our experimental setting, which constrains the double recordings to pair of cells located in close proximity within the field of view. Notably, the coupling probability in control cultures was within the ranges reported in cultures developed in the presence of growth-promoting peptides (Segal, 1983; Kasap Varley et al., 2011).

To characterize the short-term dynamics of synaptic contacts, we delivered, to pairs of connected neurons, a series of $20 \mathrm{~Hz}$ trains $(\geq 10$ sweeps for experiment); in each sweep, peak amplitudes of PSCs were measured using as reference baseline the tail of the previous event, all values were then normalized for the first PSC peak amplitude of each train. Responses varied from short-term depression, no changes, to short-term potentiation; the average responses were obtained via pooling together all recorded pairs for the two groups of culturing conditions. It is interesting to note that, in dissociated hippocampal cultures grown in the presence of peptide layering, GABAergic synapses are known to undergo short-term depression in response to frequency stimulations (Wilcox et al., 1994; Jensen et al., 1999).

In a set of experiments, the probability of having failures during the train (lack of unitary PSC within $5 \mathrm{~ms}$ from presynaptic AP peak) was quantified within each train. For each synaptically coupled pair, the percentage of failures was measured in 10 subsequent sweeps as the rate between the total number of postsynaptic failures and total number of presynaptic stimuli. Failures in PSCs were not averaged within the analysis of the short-term plasticity.

In addition, in each pair, hyperpolarizing current steps $(-0.05 \mathrm{nA} ; 100$ $\mathrm{ms}$ ) were delivered to the presynaptic cell to check the presence of electrical synapses (Zsiros et al., 2007). According to Zsiros et al. (2007), gap junctions can be identified by the presence of currents in the postsynaptic neuron coincident to presynaptic hyperpolarizing stimuli. In all our recordings $(n=166$ pairs in control and $n=138$ pairs in CNT), we detected only a small fraction of gap-junction-coupled neurons ( $2 \%$ in control and $4 \%$ in the presence of CNT). Due to the low probability of finding gap-junction-coupled pairs, those detected were not further analyzed, and were excluded from any subsequent pair analysis.

When recording spontaneous synaptic currents, CNT neurons, in agreement with Lovat et al. (2005) and Mazzatenta et al. (2007), displayed a typical strong increase in event frequency when compared to controls. As summarized in Table 3, we found that both AMPA receptorand $\mathrm{GABA}_{\mathrm{A}}$ receptor-mediated components were boosted, in terms of frequency, by the presence of CNT.

Miniature PSC. Single-neuron voltage-clamp recordings of spontaneous synaptic activity were performed in the presence of $1 \mu \mathrm{M}$ TTX at 8-9 $\mathrm{d}$ in vitro. TTX is a well known voltage gated, fast $\mathrm{Na}^{+}$channel blocker, that inhibits AP generation. Thus, spontaneous events recorded in the presence of TTX, termed miniature PSCs (mPSCs), rely on the APindependent stochastic fusion of neurotransmitter vesicles at the presynaptic membrane and their frequency is traditionally accepted to be proportional to the number of synaptic contacts (Raastad et al., 1992). The occurrence of the AMPA mPSCs was predominant in comparison to that of $\mathrm{GABA}_{\mathrm{A}}$-mediated ones (identified as previously described). The relative lack of $\mathrm{GABA}_{\mathrm{A}}$-mediated $\mathrm{mPSC}$ in cultured hippocampal networks has been previously reported (even in the presence of growthpromoting peptides; Wilcox et al., 1994; Chudotvorova et al., 2005; Singh et al., 2006; Marchionni et al., 2009) and prevented any further quantification and reliable statistical analysis, while AMPA receptor-mediated mPSCs in CNT networks displayed a significant increase in frequency when compared to controls (in control $0.23 \pm 0.03 \mathrm{~Hz}, n=55$ cells; in CNT $0.48 \pm 0.08 \mathrm{~Hz}, n=56$ cells; $p<0.05$ ), while their mean peak amplitude value was not changed (in control $92 \pm 4 \mathrm{pA}, n=55$; in CNT $97 \pm 6 \mathrm{pA}, n=56)$. The results obtained by single-neuron voltage-clamp recordings of glutamatergic miniature spontaneous synaptic currents suggested the occurrence of a parallel increase in glutamate-mediated synaptic contacts in CNT cultured networks.

\section{Immunofluorescence staining}

Hippocampal neurons were fixed with $4 \%$ paraformaldehyde/4\% sucrose in PBS for $10 \mathrm{~min}$. Free aldehyde groups were quenched in $0.1 \mathrm{M}$ glycine in PBS for $5 \mathrm{~min}$, and the neurons were blocked in 1\% fetal bovine serum (FBS) in PBS for 30 min to prevent unspecific binding of antibodies. To label only surface $\mathrm{GABA}_{\mathrm{A}}$ receptors, neurons were incubated with (rabbit) polyclonal antibody against the $\mathrm{N}$-terminal of $\gamma 2$ subunit (1:100; Alomone Labs) for $1 \mathrm{~h}$. They were then permeabilized with $0.1 \%$ Triton 
X-100 in PBS for 2 min and blocked again in $1 \%$ FBS in PBS for $30 \mathrm{~min}$. After incubation with (mouse) monoclonal antibody against the vesicular GABA transporter (VGAT; 1:200; Synaptic Systems) for $1 \mathrm{~h}$, they were incubated with a mixture of secondary antibodies (Alexa Fluor 488 goat anti-mouse, 1:500 and Alexa Fluor 647 goat anti-rabbit, 1:500) for $45 \mathrm{~min}$. Both secondary antibodies were obtained from Invitrogen.

\section{Confocal imaging}

Confocal Imaging was performed using a Nikon Eclipse C1si Confocal Microscope. Images were acquired with a $60 \times 1.4$ NA oilimmersion objective, additionally magnified threefold, so that all the images have the same dimension. Stacks of $z$-sections with an interval of $0.2 \mu \mathrm{m}$ were sequentially scanned twice for each emission line to improve the signal/ noise ratio. Offline analysis of the confocal images was performed using Image (http:// imagej.nih.gov/ij/) and $Z$-projection of the stack of images was obtained.

\section{Analysis for colocalization}

Analyses of the cluster density of synaptic connections were performed using Meta-Morph Imaging System (Universal Imaging). $\mathrm{GABA}_{\mathrm{A}}$ receptor and VGAT intensities in the entire image were analyzed. Images were segmented to select immunofluorescent puncta over background labeling, and clusters were defined as $>3$ pixels as determined by visual inspection. Integrated Morphometry Analysis function of MetaMorph was used to quantify the number of clusters. For the analysis of colocalization, a binary mask of the segmented image for each channel was created and overlapping regions with $>2$ pixels were defined as colocalized. Seven to ten images randomly selected for each culture series (control and CNT neurons; three different culture series) were quantified.

\section{Statistics}

To compare datasets, parametric and nonparametric tests (Student's $t$ test or KolmogorovSmirnov test) were used, and $p<0.05$ was considered significant, unless otherwise indicated. Tests for normality and comparisons of distributions were made using KolmogorovSmirnov tests. Values in text represent mean \pm SEM for normally distributed data, where $n=$ number of cells, unless otherwise indicated. For measurement of the PSC amplitude ratio, at least 10 consecutives sweeps were averaged. Therefore, also the steady-state response during trains was determined as the average of $\geq 50$ responses.

\section{Results}

\section{Characterization of carbon nanotube scaffolds}

Carbon nanotubes can be obtained by means of different production procedures (arc discharge, laser ablation, and chemical vapor deposition), which may lead to SWCNT or MWCNT production, depending on the temperature and gas pressure used during the process. Such procedures may affect carbon nanotube impact on biological systems in general and on neurons, in particular. In addition, several postsynthetic approaches may indirectly mediate the biological effects of carbon nanotubes, together with their variable dimensions, lengths, number of walls, degrees of purity, and metal
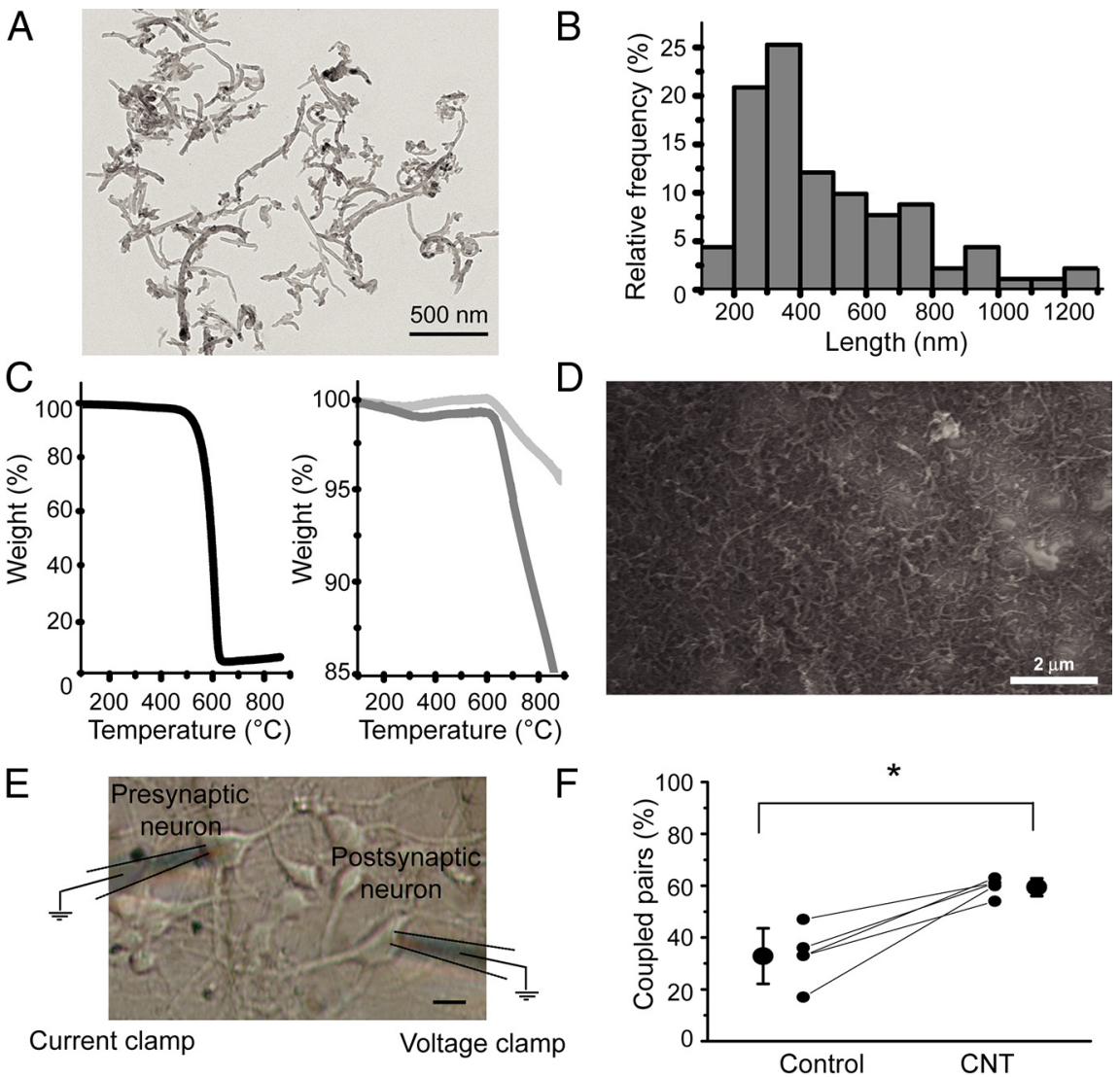

Figure 2. Physical-chemical characterization of MWCNT substrates and their impact on hippocampal network connectivity. $\boldsymbol{A}_{\text {, }}$ TEM image of functionalized MWCNTs. The image shows the good dispersibility of MWCNTs in dimethylformamide (DMF). $\boldsymbol{B}$, Length distribution plot of MWCNTs. The average length ( $480 \pm 26 \mathrm{~nm} ; n=91)$ of a sample of individualized MWCNTs was measured by processing TEM images. C, TGA analyses under nitrogen (left) and under air (right). TGA analysis provides information sphere), as a function of the high temperature. In the first plot (left), the black curve shows the metallic content of MWCNTs. In the second plot (right), the dark-gray curve represents functionalized MWCNTs, with an amount of $0.062 \mathrm{mmol} / \mathrm{g}$ of functional groups. bstrate ready for neuronal cell growth. After depositing MWCNTs on the coverslips, the substrates were annealed and subsetly SEM imaged to assess the integrity of MWCNTs after defunctionalization. Note the continuity of the carbon nanotube film is stimulated (under current-clamp configuration, left) to fire APs. The presence of monosynaptic connections to the ercentage of monosynaptically coupled pairs, is shown (small filled dots) for five different culture series (between 11 and 26 pairs, in both control and in (NT neurons, were recorded from $\geq 10$ coverslips in each series). In each culture, the coupling probability is higher for neurons grown on MWCNTs when compared to that of controls. The difference in mean values, reported as larger dots laterally, is statistically significant ( $n=85$ and $n=87$ pairs, in control and in CNT, respectively; ${ }^{*} p<0.05$ ).

content. Given all the variables in the synthesis, purification and functionalization of carbon nanotubes, we performed a first set of experiments to provide an accurate chemical-physical characterization of MWCNT supports, before their use in neurobiological applications.

This assessment was also crucial to confirm the method used, to test every new batch of MWCNTs and to avoid having misleading evaluations of the neurobiological effects emerging from MWCNTs not properly purified (Cellot et al., 2010). In fact, regardless the accepted great potential of carbon nanotubes, their widespread adoption for biological and medical applications requires a cautious approach due to their potential toxicity to the environment and human health (Kotov et al., 2009; Sucapane et al., 2009; Cellot et al., 2010). 


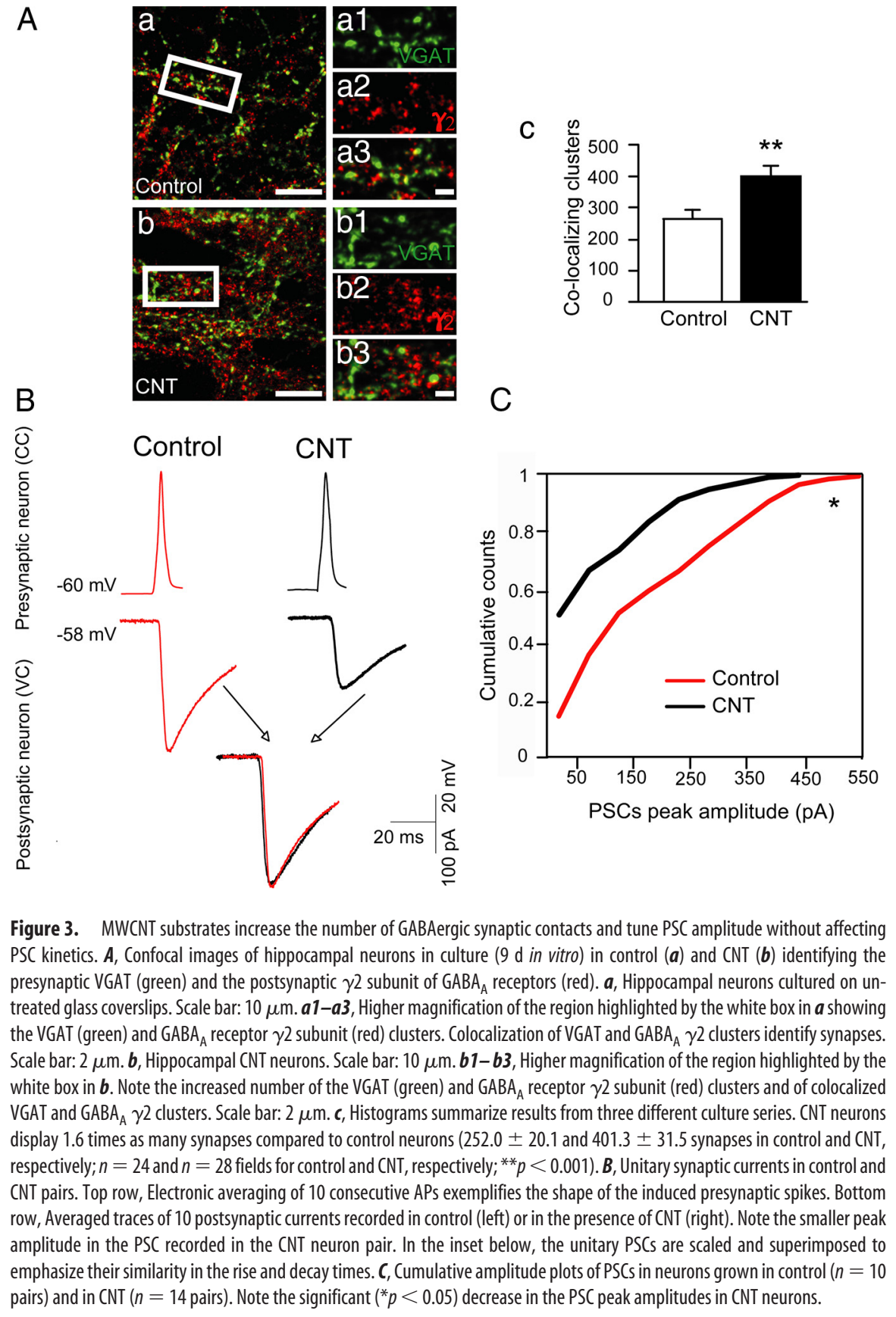

After functionalizing MWCNTs with a 1,3-dipolar cycloaddition (Georgakilas et al., 2002; Lovat et al., 2005; Mazzatenta et al., 2007; Cellot et al., 2009), we obtained a carbon nanotube solution and deposited it onto glass coverslips in controlled amounts (see Materials and Methods). Before film deposition, the nanotubes were characterized using TEM and TGA. The TEM analysis (Fig. $2 A$ ) indicated a typical dispersion of MWCNTs with a diameter range of $20-30 \mathrm{~nm}$. The MWCNT lengths (Fig. $2 B$ ) were distributed in the $100-1250 \mathrm{~nm}$ range. These values are below those that have been reported to induce serious inflammatory responses after abdominal administration of MWCNT solutions in rodents (Poland et al., 2008). Another equally important characteristic (in terms of toxicity) is the carbon nanotube metal content (tested in cell lines, Vittorio et al., 2009); TGA under air (Fig. 2C, left) indicated that this value was $<4 \%$ in our samples. After deposition, the MWCNT layers were defunctionalized by annealing under inert at- mosphere, and we further assessed the efficiency of this treatment using both TGA (Fig. $2 C$, right plot) and SEM (Fig. 2D). As indicated by Figure $2 D$, the carbon nanotube layer displayed a homogenous distribution that resulted in a large continuous carpet for the growth of brain explants. The mean roughness of the purified MWCNT film, measured by AFM (see Fig. 1), was $30.7 \pm 4.0 \mathrm{~nm}$. This value allows the occurrence of tight contacts between the neuronal membranes and the substrate (Silva, 2006; Cellot et al., 2009). To complete the MWCNT layer characterization, the film conductivity was extrapolated using MWCNT sheet resistance and AFM measurements, and was calculated to be $3.82 \times 10^{5} \mathrm{~S} / \mathrm{m}$ (Wu et al., 2004).

We used these substrates to grow rat dissociated hippocampal cultures with the aim of investigating the impact of carbon nanotubes on synapse formation and function.

Carbon nanotube scaffolds enhance the formation of GABAergic synapses

To determine how nanoscaffolds influence synapses, we examined and compared neuronal networks sustained by nanotube meshwork with those grown on a control substrate (named CNT and control, respectively). The electrophysiological properties of the synapses were measured using simultaneous whole-cell recordings from pairs of interconnected neurons (Fig. 2E). Paired recordings allow a detailed examination of the evoked transmissions of isolated synaptically coupled neurons of a known type. APs were evoked in the presynaptic cell and the resulting $\mathrm{GABA}_{\mathrm{A}}$ receptor-mediated (see Materials and Methods) unitary PSCs were examined in the postsynaptic element (PSC delay $\sim 2 \mathrm{~ms}$; Table 1 ).

Figure $2 F$ reports the percentage of cell pairs that exhibited a detectable unitary connection (coupled pairs) for controls and CNT networks. The coupling probability was significantly increased (from $33 \pm 5 \%$ to $60 \pm 2 \%$; $p<0.05$ ) by the presence of carbon nanotubes, with a connectivity ratio $(\mathrm{CR}=\mathrm{CNT}$ coupling probability/control coupling probability) of 1.8 , while the neuronal density was unaffected (see Materials and Methods). This finding is in agreement with our previous results (Lovat et al., 2005; Mazzatenta et al., 2007; Cellot et al., 2009), in which MWCNT or SWCNT substrates strongly increased the frequency of spontaneous synaptic events, and it suggests that an enhanced number of synaptic contacts are constructed under the guidance of nanotube layers in networks of similar size.

To estimate whether changes in synaptic density may account for the enhanced coupling probability observed in CNT neurons, cultured hippocampal neurons were coimmunostained for the $\gamma 2$ subunits containing $\mathrm{GABA}_{\mathrm{A}}$ receptors and for VGAT, the vesicular GABA transporter considered a GABAergic presynaptic 
marker (Dumoulin et al., 2000). The putative colocalization of presynaptic VGAT and postsynaptic $\gamma 2$ (examined in double staining experiments and quantified by confocal analysis) reliably indicates a GABAergic connection (Dumoulin et al., 2000). As shown in Figure $3 A$, a significant increase in VGAT immunoreactivity was found in CNT neurons stained for $\gamma 2$ (Fig. 3Ab-b3) indicating an enhancement of GABAergic innervation (the average number of colocalized clusters was $252.0 \pm 20.1$ and $401.3 \pm 31.5$, in control and CNT neurons, respectively; $p<$ 0.001 ; Fig. $3 A$, histograms in $c$ ) when compared to controls (Fig. 3Aa-a3).

The increased GABAergic innervation was associated with a significant reduction in amplitude of $\mathrm{GABA}_{\mathrm{A}}$-mediated PSCs (on average, the peak amplitude of PSC was $188 \pm 13 \mathrm{pA}$ and $93 \pm 8 \mathrm{pA}$, in controls and in CNT, respectively; Fig. $3 B$ ) in the absence of any effect on success rate, latency, rise, and decay time values (see summary in Table 1). In addition, as shown in the cumulative plot of Figure $3 C$, peak PSC amplitudes for control pairs were distributed to the right of CNT (the two curves were significantly different; $p<0.05)$.

\section{Carbon nanotube scaffolds remove GABAergic short-term depression}

A striking feature of neuronal transmission is the short-term dynamics of synaptic connections that governs their processing abilities (Zucker, 1989); that is, the synapses may display a transient alter-

ation in strength when activated repetitively. To assess whether carbon nanotube scaffolds affect $\mathrm{GABA}_{\mathrm{A}}$-mediated short-term plasticity, in a second set of experiments trains of six spikes at 20 $\mathrm{Hz}$ were elicited in presynaptic neurons and repeated 10 times at $10 \mathrm{~s}$ intervals. As indicated by Figure $4 \mathrm{~A}$, in controls, the amplitude of GABAergic currents became progressively smaller (the amplitude of the sixth PSC was $40 \pm 4 \%$ of the first one; Fig. $4 A, B$ ), indicating short-term synaptic depression (Abbott et al., 1997). This depression was associated with $17 \pm 7 \%$ of failures in subsequent PSCs during the train ( $n=12$ pairs).

In contrast, in CNT neurons the amplitude of $\mathrm{GABA}_{\mathrm{A}^{-}}$ mediated PSC remained stable, or slightly potentiated (on average, the amplitude of the sixth PSC was $86 \pm 18 \%$ of the first one; Fig. $4 A, B$ ), without PSC failures during the train response. This is further quantified in Figure $4 C$, in which the cumulative plots of PSC peak amplitudes obtained in CNT pairs were distributed to the right of those of control pairs (a statistically significant difference; $p<0.05)$.

Changes in short-term synaptic depression between controls and CNT neurons may underline modifications in the probability of GABA release. To further address this point, we changed the probability of release in CNT neurons by changing the extracellular $\mathrm{Ca}^{2+}$ concentration. By increasing the $\left[\mathrm{Ca}^{2+}\right]_{\mathrm{o}}$ from $2 \mathrm{mM}$ to $2.5 \mathrm{~mm}$, we increased the amplitude of the first PSC (from 42 $\mathrm{pA}$ to $51 \mathrm{pA}$; superimposed blue trace in Fig. $4 D$, right) and
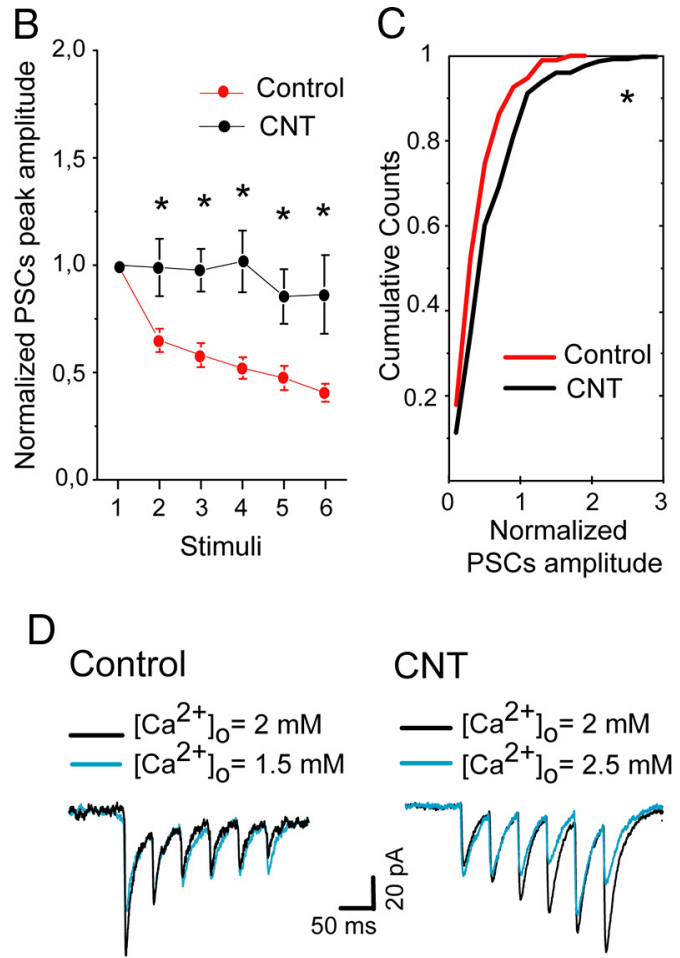

Figure 4. MWCNTs growth substrates affect short-term synaptic plasticity. $\boldsymbol{A}$, Train of six current pulses at $20 \mathrm{~Hz}$ evoked (black line) were analyzed for control and (NT eurons, respectively. D, Calcium dependence of short-term depression, in control, and of short-term potentiation, in CNT neurons. Average traces (4 sweeps) of train stimulation from a control pair (left) and a CNT one (right) for two different $\left[\mathrm{Ca}^{2+}\right]_{0}$. Note the changes in the amplitude of the first and the sixth PSC of the train when the standard [ $\left.\mathrm{Ca}^{2+}\right]_{0}$ (black traces) is decreased (control, left) or increased (CNT, right) (superimposed blue traces).

abolished the short-term potentiation, suggesting an increase in the probability of GABA release (the amplitude of the sixth PSC changed from $219 \%$ to $84 \%$ of the first; Fig. $4 D$, right). Accordingly, decreasing the $\left[\mathrm{Ca}^{2+}\right]_{\mathrm{o}}$ from $2 \mathrm{~mm}$ to $1.5 \mathrm{~mm}$ in a control pair, reduced the amplitude of the first PSC (from $107 \mathrm{pA}$ to 73 pA; superimposed blue trace in Fig. $4 D$, left) and, concomitantly, reduced the extent of short-term synaptic depression (the amplitude of the sixth PSC varied from $20 \%$ to $47 \%$ of the first; Fig. $4 D$, left). Data were confirmed in $n=3$ control and $n=3$ CNT pairs. These results indicate the involvement of release-dependent mechanisms in the shifting dynamics of CNT synapses (Dittman and Regehr, 1998; Dittman et al., 2000).

\section{Carbon nanotube-driven changes in synaptic dynamics depend on network activity and spike propagation}

In our previous work (Cellot et al., 2009), we have shown that the conductivity of carbon nanotubes, together with their direct junctions to neurons, favor the generation of back-propagating APs which in turn may contribute to synaptic dynamics. Backpropagating APs are involved in spike-time-dependent plasticity (Markram et al., 1997; Dan and Poo, 2004), in the regulation of local synaptic feedback, and in the $\mathrm{Ca}^{2+}$-dependent release of messengers, ultimately controlling activity-dependent changes in synaptic efficacy (Waters et al., 2005; Zilberter et al., 2005; Kuczewski et al., 2008). In principle, promoting the back- 
A
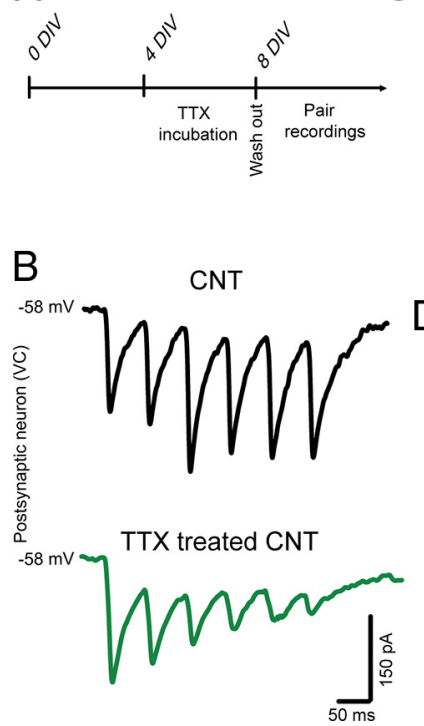

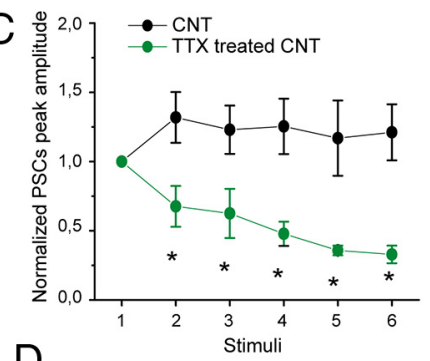

$\mathrm{D}$

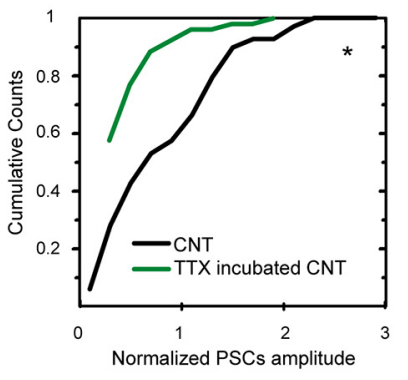

Figure 5. TTX chronic incubation removes MWCNT substrate changes in PSC short-term plasticity. $A$, Chronic removal of APs is achieved by incubating cultures with TTX for $4-5 \mathrm{~d}$. At $8-9 \mathrm{~d}$ in vitro, upon $2 \mathrm{~h}$ of TTX washout, pair recordings are performed. $\boldsymbol{B}$, Averaged traces are obtained by delivering 10 consecutive trains of stimuli ( 6 pulses at $20 \mathrm{~Hz}$ ) in untreated (above) and TTX-treated (below) neuronal pairs of sister CNT cultures. Note the appearance of shortterm depression in TTX chronically treated CNT cultures. C, The plot summarizes results from different neuronal pairs, taken from untreated and TTX-treated sister CNT cultures. In each neuron, PSC amplitudes are normalized to that of the first PSC of the train. Recordings are from a sample of six pairs of untreated CNT (black dots) neurons and from seven pairs of TTX-treated CNT neurons (green dots). Note the statistically significant $\left({ }^{*} p<0.05\right)$ differences. $D$, Significant $\left({ }^{*} p<0.05\right)$ differences in the short-term dynamics of monosynaptic PSCs are indicated by the shift in the cumulative amplitude plots of PSC (constructed as in Fig. 4). Seven pairs (black line) and six pairs (green line) were analyzed for untreated and TTX-treated CNT neurons, respectively.

propagation of APs could contribute to the carbon nanotubeinduced changes in synaptic dynamics.

To test whether the nanotube-driven changes in synaptic function required network activity and spike propagation, control and CNT neurons were chronically exposed (for 4-5 d) to $1 \mu \mathrm{M}$ TTX (see Materials and Methods and sketch in Fig. $5 \mathrm{~A}$ for the experimental design). After chronic treatment (followed by TTX washout), we performed standard dual electrophysiological recordings from both control and CNT neurons to compare TTX-treated and untreated neurons. Also in the case of the TTX-treated cultures, recorded pairs displayed GABAergic synapses in the large majority $(\geq 80 \%)$ of the monosynaptically coupled neurons, exhibiting features in the presynaptic and postsynaptic signals that were similar to those measured in untreated samples (Table 2).

TTX treatment did not modify the CNT-induced increase in the CR (1.9 in TTX-treated vs 1.8 in untreated cultures; $n=10$ and $n=25$ pairs, control and CNT, respectively), but it converted the CNT-induced short-term synaptic potentiation into shortterm depression.

When trains of APs were evoked, chronic deprivation of network activity caused a reversion of the changes induced by carbon nanotubes in the synaptic frequency-dependent dynamics. The sample tracings of Figure $5 B$ indicate that CNT synapses undergo short-term depression upon TTX treatment, just as the control synapses do (the plot in Fig. 5C summarizes data from pooled untreated and TTX-treated CNT pairs). This is further quantified in Figure 5D, in which the cumulative plots of PSC peak amplitudes obtained in CNT pairs were distributed to the right of those

of TTX-treated CNT pairs (a statistically significant difference; $p<0.05)$. Conversely, TTX-treatment of the control cultures did not affect the amplitude of the first PSC, nor did it affect the dynamics of the synaptic responses, which retained their shortterm depression (the amplitude of the sixth PSC changed from $52 \pm 13 \%$ to $30 \pm 7 \%$ of the first, respectively untreated control cultures, $n=10$ and TTX-treated ones, $n=11$ ).

\section{Discussion}

The results presented here provide new knowledge about the functional interactions between neuronal networks and nanoscaled growth supports. Our study reveals for the first time that (1) carbon nanotubes are able to promote an AP-independent increase in the global connectivity of hippocampal networks and (2) the synapses that are newly formed in the presence of carbon nanotubes display different short-term synaptic dynamics, and the expression of such a change requires network activity and spike propagation.

MWCNT scaffolds may lead to the increased connectivity by promoting synaptogenesis. This could be achieved either directly (providing unnatural biomimetic cues) or indirectly (modulating the deposition of an extracellular matrix, ECM, more permissive for synapse construction, Berardi et al., 2004).

Nanomaterial's direct impact on cell assemblies, in engineered tissue scaffolds, is currently used to compensate for the low strength or low electrical conductivity of biomaterials (Dvir et al., 2011). In addition, topographical features expressed by the incorporated nanomaterials, together with their physical and chemical properties, may provide to neurons a wealth of information (Place et al., 2009; Dvir et al., 2011). Direct interactions, due to the formation of spontaneous tight junctions, as described in carbon nanotube hybrids with neuronal membranes (Cellot et al., 2009) or with lipid bilayers (Zhou et al., 2007), may influence mechanical forces involved in adhesion processes and, ultimately, all these factors may affect synapse stabilization.

Alternatively, MWCNT nanoscaffolds may influence cell assemblies indirectly, via a control of the ECM deposition. It has been shown that cultured neurons are able to construct perineuronal net (PNN)-like structures (Miyata et al., 2005; Dityatev et al., 2007; Frischknecht et al., 2009). We may speculate that MWCNT scaffolds alter ECM deposition in cultured neurons, ultimately influencing synaptogenesis (Berardi et al., 2004).

To distinguish between these two hypotheses requires further investigations, beyond the present one, and requires on one hand the design of experiments using specific tools, such as AFM in liquid environment, addressing the membrane adhesion at the nanoscale, and on the other that of experiments designed to compare the distribution and formation of PNN in the two culture groups, and linking such changes to electrophysiological recordings.

An alternative possibility is that the detected improved connectivity may be due to the presence of MWCNT-mediated shortcuts at the network level, artificially and directly connecting neurons, similar to those hypothesized at the single-cell level (artificially connecting distal neuronal compartments; Cellot et al., 2009). The low occurrence of direct electrical connections between pairs of neurons belonging to the two groups of cultures, control and CNT, seems to exclude this possibility.

In CNT networks, the increased connectivity contributes in boosting spontaneous synaptic activity, leading to an increase in PSC frequency, which may in turn affect the adaptability of the network (Ivenshitz and Segal, 2010). In this respect, the smaller amplitudes of the evoked unitary PSCs in CNT networks may be due to a general homeostatic optimization of synaptic strength as 
a function of the number of synaptic contacts and activity (Ivenshitz and Segal, 2010; Pozo and Goda, 2010).

The amplitude of unitary synaptic currents is regulated by several presynaptic and postsynaptic factors (Atwood and Karunanithi, 2002; Schneggenburger et al., 2002; Zucker and Regehr, 2002). Unfortunately, the virtual absence of GABAergic miniature PSCs (see Materials and Methods) did not allow investigation of the potential contribution of postsynaptic changes or quantification of the GABA quantal content.

Nonetheless, in our experiments, the reduced PSC amplitude observed in CNT synapses seems to reflect a lowered probability of GABA release. This is strengthened by the reported ability of carbon nanotubes to influence short-term synaptic plasticity, inducing a substantial reversion of short-term depression at CNT synapses, and by the reported dependence of short-term plasticity from the $\left[\mathrm{Ca}^{2+}\right]_{\mathrm{o}}$ (Atwood and Karunanithi, 2002; but see also Zucker and Regehr, 2002). Thus, due to a negative feedback process, did the persistent elevation in synaptic contacts in CNT neuronal circuits decrease presynaptic release at CNT synapses, ultimately leading to local changes in short-term plasticity? Against a straight compensatory hypothesis, we observed that, after chronic TTX treatment, CNT pairs maintain the usual CR increase, but unitary PSCs tend to show larger amplitudes, accompanied by short-term depression. On the other hand, AP removal per se did not affect control neurons.

It might not be a coincidence that carbon nanotubes were not able to induce synaptic plastic changes in the absence of APs. We recently reported that carbon nanotube growth substrates favor the generation of back-propagating APs (Cellot et al., 2009). At the time, we could not establish whether enhanced dendritic regenerative properties had a role in regulating synaptic contacts. Now we know that the increased connectivity brought about by carbon nanotubes does not rely on such a phenomenon. However, improved dendritic regenerative properties in CNT neurons may be responsible for synaptic dynamics tuning.

It is tempting to speculate, that carbon nanotube-induced changes in synaptic dynamics are not a mere compensation of the increased connectivity. Rather, the specificity of conductive carbon nanotube/neuron interactions, by favoring the back propagation of APs (Cellot et al., 2009), allows neurons to interpret substrates and to tune their synaptic activity. The ability of carbon nanotubes to interact with neuronal processes may help us to understand the normal physiological mechanisms, such as backpropagating APs in real neuronal networks, that are involved in the modulation of core synaptic properties.

Our novel findings may lead to the exploitation of artificial submicroscopic man-designed devices that cooperate to neuronal network activity, generating hybrid structures able to cross the barriers between artificial devices and neurons.

The development of hybrid neuronal-nanomaterial networks holds the potential to improve our knowledge on the adhesive interactions that cells are able to probe and respond to. This innovative technology will potentially allow addressing key factors in the cellular microenvironment able to influence cell behavior via a chemical and physical code. This system can be used as a platform to examine cell detection of, and reactions to, environmental physical features. In addition, hybrids able to boost synaptic performance may be exploited to test information processing in neuronal networks.

\section{References}

Abbott LF, Varela JA, Sen K, Nelson SB (1997) Synaptic depression and cortical gain control. Science 275:220-224.
Atwood HL, Karunanithi S (2002) Diversification of synaptic strength: presynaptic elements. Nat Rev Neurosci 3:497-516.

Avossa D, Rosato-Siri MD, Mazzarol F, Ballerini L (2003) Spinal circuits formation: a study of developmentally regulated markers in organotypic cultures of embryonic mouse spinal cord. Neuroscience 122:391-405.

Basarsky TA, Parpura V, Haydon PG (1994) Hippocampal synaptogenesis in cell culture: developmental time course of synapse formation, calcium influx, and synaptic protein distribution. J Neurosci 14:6402-6411.

Berardi N, Pizzorusso T, Maffei L (2004) Extracellular matrix and visual cortical plasticity: freeing the synapse. Neuron 44:905-908.

Bi GQ, Poo MM (1998) Synaptic modifications in cultured hippocampal neurons: dependence on spike timing, synaptic strength, and postsynaptic cell type. J Neurosci 18:10464-10472.

Bolton MM, Pittman AJ, Lo DC (2000) Brain-derived neurotrophic factor differentially regulates excitatory and inhibitory synaptic transmission in hippocampal cultures. J Neurosci 20:3221-3232.

Cellot G, Cilia E, Cipollone S, Rancic V, Sucapane A, Giordani S, Gambazzi L, Markram H, Grandolfo M, Scaini D, Gelain F, Casalis L, Prato M, Giugliano M, Ballerini L (2009) Carbon nanotubes might improve neuronal performance by favouring electrical shortcuts. Nat Nanotechnol 4: $126-133$.

Cellot G, Ballerini L, Prato M, Bianco A (2010) Neurons are able to internalize soluble carbon nanotubes: new opportunities or old risks? Small 6:2630-2633

Chudotvorova I, Ivanov A, Rama S, Hübner CA, Pellegrino C, Ben-Ari Y, Medina I (2005) Early expression of KCC2 in rat hippocampal cultures augments expression of functional GABA synapses. J Physiol 566:671-679.

Cui H, Webber MJ, Stupp SI (2010) Self-assembly of peptide amphiphiles: from molecules to nanostructures to biomaterials. Biopolymers 94:1-18.

Dan Y, Poo MM (2004) Spike timing-dependent plasticity of neural circuits. Neuron 44:23-30.

Dittman JS, Regehr WG (1998) Calcium dependence and recovery kinetics of presynaptic depression at the climbing fiber to Purkinje cell synapse. J Neurosci 18:6147-6162.

Dittman JS, Kreitzer AC, Regehr WG (2000) Interplay between facilitation, depression, and residual calcium at three presynaptic terminals. J Neurosci 20:1374-1385.

Dityatev A, Brückner G, Dityateva G, Grosche J, Kleene R, Schachner M (2007) Activity-dependent formation and functions of chondroitin sulfate-rich extracellular matrix of perineuronal nets. Dev Neurobiol 67:570-588.

Dumoulin A, Lévi S, Riveau B, Gasnier B, Triller A (2000) Formation of mixed glycine and GABAergic synapses in cultured spinal cord neurons. Eur J Neurosci 12:3883-3892.

Dvir T, Timko BP, Kohane DS, Langer R (2011) Nanotechnological strategies for engineering complex tissues. Nat Nanotechnol 6:13-22.

Ellis-Behnke RG, Liang YX, You SW, Tay DK, Zhang S, So KF, Schneider GE (2006) Nano neuro knitting: peptide nanofiber scaffold for brain repair and axon regeneration with functional return of vision. Proc Natl Acad Sci U S A 103:5054-5059.

Frischknecht R, Heine M, Perrais D, Seidenbecher CI, Choquet D, Gundelfinger ED (2009) Brain extracellular matrix affects AMPA receptor lateral mobility and short-term synaptic plasticity. Nat Neurosci 12:897-904.

Galante M, Nistri A, Ballerini L (2000) Opposite changes in synaptic activity of organotypic rat spinal cord cultures after chronic block of AMPA/ kainate or glycine and GABAA receptors. J Physiol 523:639-651.

Galvan-Garcia P, Keefer EW, Yang F, Zhang M, Fang S, Zakhidov AA, Baughman RH, Romero MI (2007) Robust cell migration and neuronal growth on pristine carbon nanotube sheets and yarns. J Biomater Sci Polym Ed 18:1245-1261.

Geller HM, Fawcett JW (2002) Building a bridge: engineering spinal cord repair. Exp Neurol 174:125-136.

Georgakilas V, Voulgaris D, Vázquez E, Prato M, Guldi DM, Kukovecz A, Kuzmany H (2002) Purification of HiPCO carbon nanotubes via organic functionalization. J Am Chem Soc 124:14318-14319.

Hu H, Ni Y, Mandal SK, Montana V, Zhao B, Haddon RC, Parpura V (2005) Polyethyleneimine functionalized single-walled carbon nanotubes as a substrate for neuronal growth. J Phys Chem B 109:4285-4289.

Ivenshitz M, Segal M (2010) Neuronal density determines network connec- 
tivity and spontaneous activity in cultured hippocampus. J Neurophysiol 104:1052-1060.

Jensen K, Lambert JD, Jensen MS (1999) Activity-dependent depression of GABAergic IPSCs in cultured hippocampal neurons. J Neurophysiol 82:42-49.

Kasap Varley Z, Pizzarelli R, Antonelli R, Stancheva SH, Kneussel M, Cherubini E, Zacchi P (2011) Gephyrin regulates GABAergic and glutamatergic synaptic transmission in hippocampal cell cultures. J Biol Chem 286:20942-20951.

Keefer EW, Botterman BR, Romero MI, Rossi AF, Gross GW (2008) Carbon nanotube coating improves neuronal recordings. Nat Nanotechnol 3:434-439.

Kotov NA, Winter JO, Clements IP, Jan E, Timko BP, Campidelli S, Pathak S, Mazzatenta A, Lieber CM, Prato M, Bellamkonda RV, Silva GA, Shi Kam NW, Patolsky F, Ballerini L (2009) Nanomaterials for neural interfaces. Adv Mater 21:3970-4004.

Kuczewski N, Porcher C, Ferrand N, Fiorentino H, Pellegrino C, Kolarow R, Lessmann V, Medina I, Gaiarsa JL (2008) Backpropagating action potentials trigger dendritic release of BDNF during spontaneous network activity. J Neurosci 28:7013-7023.

Lovat V, Pantarotto D, Lagostena L, Cacciari B, Grandolfo M, Righi M, Spalluto G, Prato M, Ballerini L (2005) Carbon nanotube substrates boost neuronal electrical signaling. Nano Lett 5:1107-1110.

Marchionni I, Kasap Z, Mozrzymas JW, Sieghart W, Cherubini E, Zacchi P (2009) New insights on the role of gephyrin in regulating both phasic and tonic GABAergic inhibition in rat hippocampal neurons in culture. Neuroscience 164:552-562.

Markram H, Lübke J, Frotscher M, Sakmann B (1997) Regulation of synaptic efficacy by coincidence of postsynaptic APs and EPSPs. Science 275:213-215.

Mattson MP, Haddon RC, Rao AM (2000) Molecular functionalization of carbon nanotubes and use as substrates for neuronal growth. J Mol Neurosci 14:175-182.

Mazzatenta A, Giugliano M, Campidelli S, Gambazzi L, Businaro L, Markram H, Prato M, Ballerini L (2007) Interfacing neurons with carbon nanotubes: electrical signal transfer and synaptic stimulation in cultured brain circuits. J Neurosci 27:6931-6936.

Melnick IV, Chvanov MA, Belan PV (1999) Rat hippocampal neurons maintain their own GABAergic synaptic transmission in culture. Neurosci Lett 262:151-154.

Miyata S, Nishimura Y, Hayashi N, Oohira A (2005) Construction of perineuronal net-like structure by cortical neurons in culture. Neuroscience 136:95-104.

Ni Y, Hu H, Malarkey EB, Zhao B, Montana V, Haddon RC, Parpura V (2005) Chemically functionalized water soluble single-walled carbon nanotubes modulate neurite outgrowth. J Nanosci Nanotechnol 5:1707-1712.

Orive G, Anitua E, Pedraz JL, Emerich DF (2009) Biomaterials for promoting brain protection, repair and regeneration. Nat Rev Neurosci 10:682-692.

Pavlidis P, Montgomery J, Madison DV (2000) Presynaptic protein kinase activity supports long-term potentiation at synapses between individual hippocampal neurons. J Neurosci 20:4497-4505.

Place ES, Evans ND, Stevens MM (2009) Complexity in biomaterials for tissue engineering. Nat Mater 8:457-470.

Plant GW, Woerly S, Harvey AR (1997) Hydrogels containing peptide or aminosugar sequences implanted into the rat brain: influence on cellular migration and axonal growth. Exp Neurol 143:287-299.

Poland CA, Duffin R, Kinloch I, Maynard A, Wallace WA, Seaton A, Stone V,
Brown S, Macnee W, Donaldson K (2008) Carbon nanotubes introduced into the abdominal cavity of mice show asbestos-like pathogenicity in a pilot study. Nat Nanotechnol 3:423-428.

Potthoff O, Dietzel ID (1997) Thyroid hormone regulates $\mathrm{Na}^{+}$currents in cultured hippocampal neurons from postnatal rats. Proc Biol Sci 264:367-373.

Pozo K, Goda Y (2010) Unraveling mechanisms of homeostatic synaptic plasticity. Neuron 66:337-351.

Raastad M, Storm JF, Andersen P (1992) Putative single quantum and single fibre excitatory postsynaptic currents show similar amplitude range and variability in rat hippocampal slices. Eur J Neurosci 4:113-117.

Schneggenburger R, Sakaba T, Neher E (2002) Vesicle pools and short-term synaptic depression: lessons from a large synapse. Trends Neurosci 25:206-212.

Segal M (1983) Rat hippocampal neurons in culture: responses to electrical and chemical stimuli. J Neurophysiol 50:1249-1264.

Shoval A, Adams C, David-Pur M, Shein M, Hanein Y, Sernagor E (2009) Carbon nanotube electrodes for effective interfacing with retinal tissue. Front Neuroeng 2:1-8.

Silva GA (2006) Neuroscience nanotechnology: progress, opportunities and challenges. Nat Rev Neurosci 7:65-74.

Silva GA (2009) Nanomedicine: shorting neurons with nanotubes. Nat Nanotechnol 4:82-83.

Singh B, Henneberger C, Betances D, Arevalo MA, Rodríguez-Tébar A, Meier JC, Grantyn R (2006) Altered balance of glutamatergic/GABAergic synaptic input and associated changes in dendrite morphology after BDNF expression in BDNF-deficient hippocampal neurons. J Neurosci 26:7189-7200.

Sucapane A, Cellot G, Prato M, Giugliano M, Parpura V, Ballerini L (2009) Interactions between cultured neurons and carbon nanotubes: a nanoneuroscience vignette. J Nanoneurosci 1:10-16.

Vittorio O, Raffa V, Cuschieri A (2009) Influence of purity and surface oxidation on cytotoxicity of multiwalled carbon nanotubes with human neuroblastoma cells. Nanomedicine 5:424-431.

Waters J, Schaefer A, Sakmann B (2005) Backpropagating action potentials in neurones: measurement, mechanisms and potential functions. Prog Biophys Mol Biol 87:145-170.

Wilcox KS, Buchhalter J, Dichter MA (1994) Properties of inhibitory and excitatory synapses between hippocampal neurons in very low density cultures. Synapse 18:128-151.

Woo KM, Chen VJ, Ma PX (2003) Nano-fibrous scaffolding architecture selectively enhances protein adsorption contributing to cell attachment. J Biomed Mater Res A 67:531-537.

Wu Z, Chen Z, Du X, Logan JM, Sippel J, Nikolou M, Kamaras K, Reynolds JR, Tanner DB, Hebard AF, Rinzler AG (2004) Transparent, conductive carbon nanotube films. Science 305:1273-1276.

Zhou X, Moran-Mirabal JM, Craighead HG, McEuen PL (2007) Supported lipid bilayer/carbon nanotube hybrids. Nat Nanotechnol 2:185-190.

Zilberter Y, Harkany T, Holmgren CD (2005) Dendritic release of retrograde messengers controls synaptic transmission in local neocortical networks. Neuroscientist 11:334-344.

Zsiros V, Aradi I, Maccaferri G (2007) Propagation of postsynaptic currents and potentials via gap junctions in GABAergic networks of the rat hippocampus. J Physiol 578:527-544.

Zucker RS (1989) Short-term synaptic plasticity. Annu Rev Neurosci 12:13-31.

Zucker RS, Regehr WG (2002) Short-term synaptic plasticity. Annu Rev Physiol 64:355-405. 